ORIGINAL ARTICLE

\title{
The "OBS" chart: an evidence based approach to re-design of the patient observation chart in a district general hospital setting
}

\author{
M T Chatteriee, J C Moon, R Murphy, D McCrea
}

Postgrad Med J 2005;81:663-666. doi: 10.1136/pgmi.2004.031872

See end of article for authors' affiliations

Correspondence to: Dr D McCrea

Postgraduate Medical Centre, Central Middlesex Hospital, Acton Lane, Park Royal, London NW10 4NS, UK; Daniel.

McCrea@nwlh.nhs.uk

Submitted

20 December 2004

Accepted

21 February 2005

\begin{abstract}
Objective: The primary role of the patient bedside observation chart is to make clinicians aware of the deteriorating patient. Despite this, its performance has not been scrutinised. Many versions exist with different styles of data entry but the optimal format remains elusive. This paper hypothesised that chart design measurably influences function and that redesign and standardisation would improve the detection of physiological decline by clinical staff.

Design: Objective evaluation of existing charts $(n=5)$, evidence based redesign, and re-evaluation of new chart.

Setting: 250 bed district general hospital.

Results: Design of existing observation charts had a significant effect on the ability of clinical staff to detect patient deterioration, with detection rates of parameters indicating physiological decline ranging from $0 \%$ to $100 \%$. Graphical plots portrayed information better than written values for all parameters being measured except tachypnoea. No single existing chart was best for all variables. A new chart was designed, implemented with training in its use, and re-evaluated. The new chart also incorporated an early warning scoring system. There were significant improvements in the average detection rates of parameters poorly identified on existing charts: detection rates of tachypnoea and hypoxia increased by $41 \%$ $(p<0.05)$ and $45 \%(p<0.05)$ respectively. There were also significant improvements in detection rates of tachycardia and fever by $29 \%(p<0.05)$ and $16 \%(p<0.05)$ respectively.

Conclusion: Evidence based redesign of the patient bedside observation chart coupled with specific training in its use significantly improves the detection of patient physiological deterioration.
\end{abstract}

$\mathrm{T}$ he identification of the deteriorating patient is the key to preventing admission to high dependency facilities. This is of particular importance on outlying medical and surgical wards where physiological decline may be highlighted late in the development of critical illness: 30\% of intensive care unit admissions receive cardiopulmonary resuscitation before intensive care admission despite being on a hospital ward, ${ }^{1}$ and up to $41 \%$ of admissions to intensive care units from wards can be avoided if earlier treatment occurs. ${ }^{2}$ Of more concern is that abnormal physiological values are often charted without action in the hours preceding an in-hospital cardiopulmonary arrest $\mathrm{t}^{3-6}$ and up to 24 hours before ward patients are admitted to intensive care. $^{7}$

One way of increasing the recognition of the high risk patient is to implement an early warning scoring system (EWS) or modified early warning scoring system (MEWS). ${ }^{8-13}$ These are algorithms that collate charted physiological data into a single risk score and are designed to alert healthcare professionals to the deteriorating patient. Mortality increases as the number of physiological abnormalities increase being as high as $21.3 \%$ with three or more abnormalities and as low as $0.7 \%$ with no abnormalities $(\mathrm{p}<0.01) .{ }^{13}$

Respiratory rate, heart rate, and adequacy of oxygenation have been implicated as indicators of critically ill patients on the general ward. ${ }^{7}$ Systolic blood pressure, temperature, and level of consciousness graded by AVPU (alert, responding to voice, responding to pain, unresponsive) are additionally predictive of adverse outcome in acute medical admissions. ${ }^{14}$

Bedside observation charts are the mainstay of detecting patient deterioration but the monitoring and charting of physiological variables is often poor. This is especially true of respiratory rate. ${ }^{1}$ Observation charts vary between and within hospitals, yet to our knowledge the effectiveness of different types of observation charts in clinical practice has not been studied. The optimal chart format thus remains elusive. Moreover, there have been no defined standards against which observations are charted.

At our institution, a 250 bed district general hospital, five charts were in use and here we test the effectiveness of different types of observation charts in highlighting physiological decline.

We hypothesise that an evidence based approach to observation chart redesign improves the detection of physiological decline by end users.

\section{METHODS}

Examples of stable and unstable physiological data were selected from ward patients and accurately plotted by a single operator across the existing five charts. These were presented in a stereotyped fashion to 63 end users in two groups: group 1, $\mathrm{n}=32$ (pre-registration house officers, senior house officers, and specialist registrars); group 2, $\mathrm{n}=31$ (healthcare assistants and student nurses: this cohort generally chart observations at our institution and clarify physiological values that concern them with a registered nurse). The ability of the various charts to allow clinical staff with different levels of training to detect unstable physiology was measured.

End users were asked to identify physiological parameters they perceived as being abnormal on the charts based on physiological parameters considered to lie within the normal range as follows: heart rate $50-100$ beats per minute; systolic blood pressure 100-160 mm $\mathrm{Hg}$; respiratory rate 10-20 per minute; oxygen saturations $>95 \%$ on air; temperature $>37^{\circ} \mathrm{C}$. 
Table 1 Detection rates (\%) of abnormal physiological parameters by doctors (normal type) and healthcare assistants and student nurses (bold) from existing charts

\begin{tabular}{|c|c|c|c|c|c|c|c|c|c|c|}
\hline Parameters measured & Chart 1 & & Chart 2 & & Chart 3 & & Chart 4 & & Chart 5 & \\
\hline Fever & 100 plotted & 100 & 100 written & 29 & 100 plotted & 65 & 100 plotted & 65 & 100 plotted & 84 \\
\hline Hypotension & 84 plotted & 87 & 100 plotted & 71 & 72 plotted & 84 & 84 plotted & 84 & 84 plotted & 84 \\
\hline Tachycardia & $\begin{array}{l}100 \text { plotted } \\
\text { with BP }\end{array}$ & 71 & 100 written & 13 & 100 written & 50 & 100 written & 50 & $\begin{array}{l}84 \text { plotted } \\
\text { with BP }\end{array}$ & 32 \\
\hline Tachypnoea & 72 plotted & 29 & 100 written & 58 & 84 written & 50 & 66 written & 32 & 84 plotted & 16 \\
\hline Hypoxia & 100 plotted & 100 & 84 written & 13 & 59 written & 16 & 84 written & 32 & 50 written & 0 \\
\hline
\end{tabular}

Results were recorded by the interviewer. Subjective chart preferences were also recorded. These data were used to design a new observation chart that also incorporated an early warning scoring system. This was standardised throughout all medical wards with clinical staff trained in its use. The new chart was re-evaluated three weeks after introduction to wards.

Statistical analysis for binomial distributions was used to assess if relative frequencies of detection of abnormal physiology were significantly different before and after chart re-design.

\section{RESULTS}

Table 1 shows the objective chart performance. Not all physiological decline was equally well detected across the five charts and there were broad ranges.

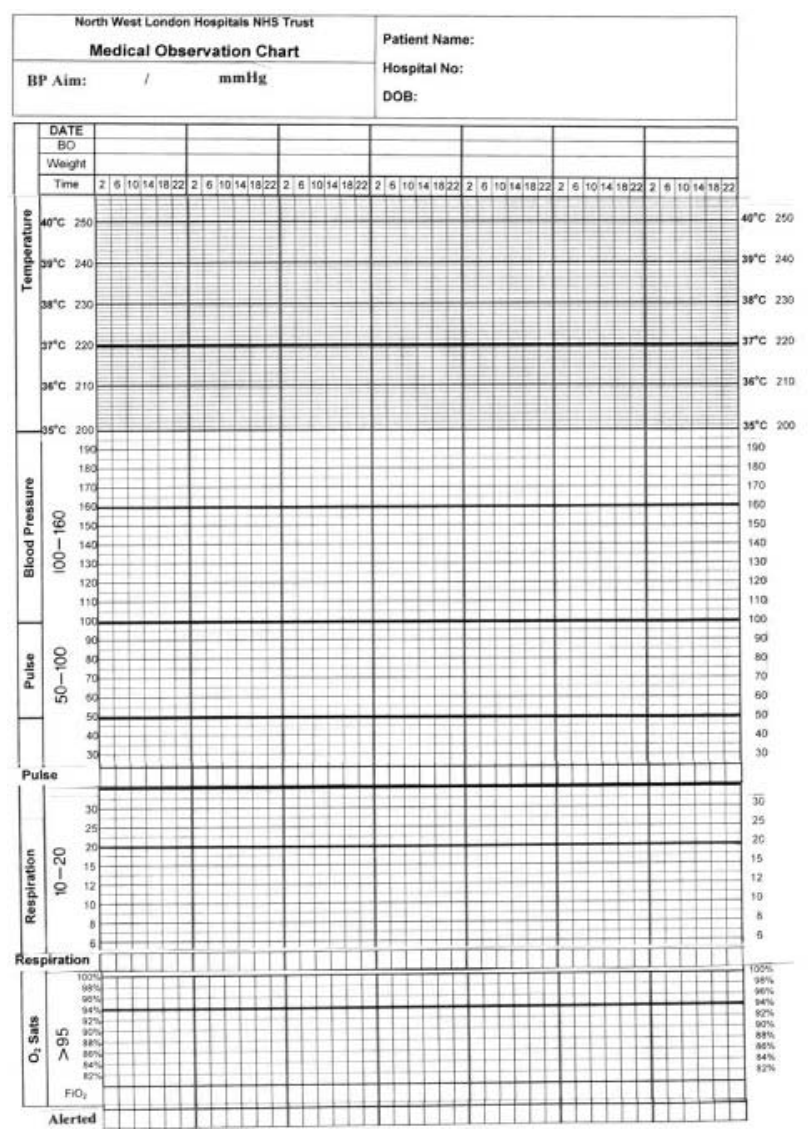

On average, tachypnoea was missed by $19 \%$ (range $0 \%-$ $34 \%$ ) of junior doctors (PRHO, SHO, SpR-cohort 1) and 63\% (range $42 \%-84 \%$ ) of healthcare assistants and student nurses (cohort 2); hypoxia by $25 \%$ (range $0 \%-50 \%$ ) of cohort 1 and $68 \%$ (range $0 \%-100 \%$ ) of cohort 2; tachycardia by 3\% (range $0 \%-16 \%$ ) of cohort 1 and $57 \%$ (range $29 \%-87 \%$ ) of cohort 2 ; hypotension by $15 \%$ (range $0 \%-28 \%$ ) of cohort 1 and $18 \%$ (range $13 \%-29 \%$ ) of cohort 2; fever was missed by $0 \%$ of cohort 1 and $31 \%$ (range $0 \%-71 \%$ ) of cohort 2 .

Combining all parameters in all charts, overall detection rates of physiological decline for cohorts 1 and 2 were $88 \%$ (range $50 \%$ to $100 \%$ ) and $53 \%$ (range $0 \%$ to $100 \%$ ) respectively.

Analysis of combined parameters showed that one chart (chart 1) at $84 \%$ had the highest average detection

Early Warning Score

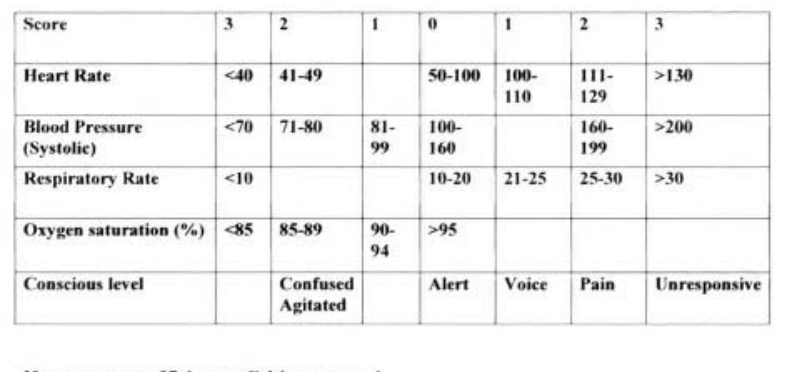

If temperature $>37$ degrees Celsius: score $=1$

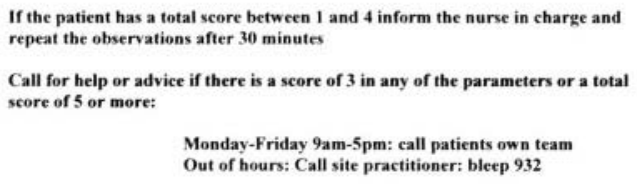

Document below: a single score of 3 or a total score of 5 or more

\begin{tabular}{|l|l|l|l|}
\hline Date \& Time & Score & Action taken & Signature \\
\hline & & & \\
\hline & & & \\
\hline & & & \\
\hline & & & \\
\hline & & & \\
\hline & & & \\
\hline & & & \\
\hline
\end{tabular}

Figure 1 Key features of new chart. Temperature-plotted on a large scale; blood pressure and heart rate plots overlain on the same axis with a separate box underneath for pulse rate to be recorded as a number - to allow for the fact that inaccurate joining of points may obscure the reading within the blood pressure plot; respiratory rate was better detected as a written parameter but duplicated on the new chart as a written number and plotted value to account for subjective preferences of clinical staff and to highlight changes in this parameter as being the most important early manifestation of critical illness; oxygen saturation plotted. Parameter values were written on both sides of the chart. A standard for detecting physiological decline-early warning score (EWS) - was incorporated on the back of the chart. Warning lines corresponding to a EWS score of 1 or 2 (first alert score for parameter) were incorporated within the chart. 
A

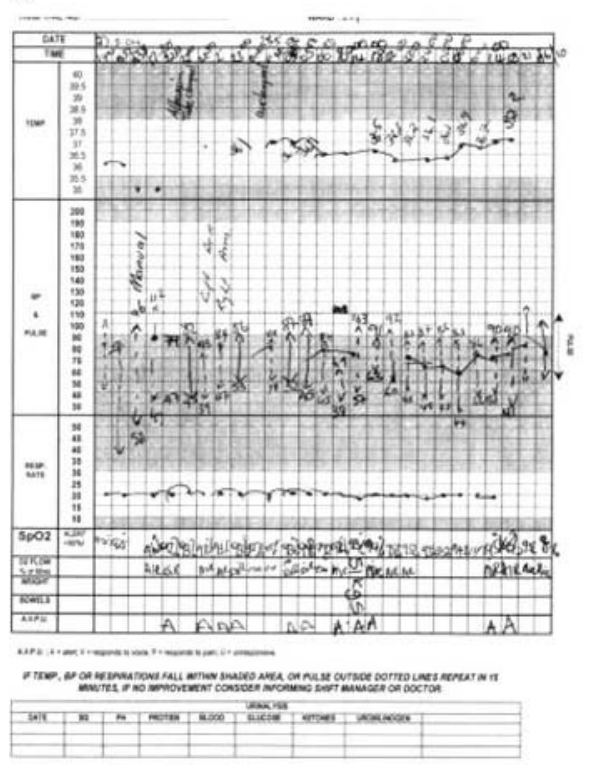

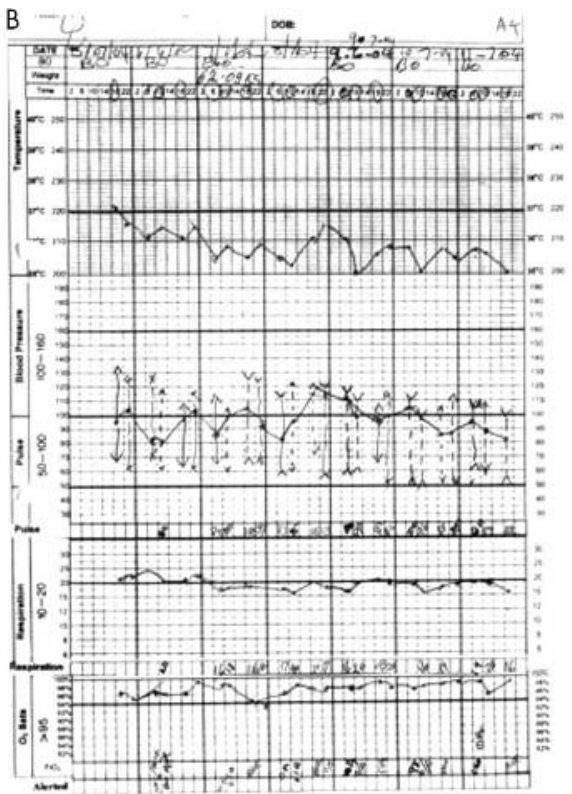

Figure 2 Examples of charts taken from the same medical ward before (A) and after (B) intervention. rates. This was $15 \%$ higher than chart $4,16 \%$ higher than chart 3, 18\% higher than chart 2, and 23\% higher than chart 5.

As well as having the highest average detection rates of physiological decline, chart 1 was also preferred by most clinical staff: $(70 \%$ of cohort 1 and $47 \%$ of cohort 2). Of the individual chart parameters, all categories were preferred on chart 1 except respiratory rate, which was preferred duplicated as a written value and charted plot.

Overall, plotted parameters were better detected than written values ( $80 \%$ compared with $58 \%$ ). Of the individual parameters, this held for fever (89\% compared with 65\%); tachycardia (72\% compared with 69\%) and hypoxia (100\% compared with $42 \%$ ). Tachypnoea was better detected when written (65\% compared with $50 \%$ ). Blood pressure readings were plotted on all charts.

A new chart was designed based on objective and subjective assessment (fig 1).

This was introduced to all wards with training in its use performed over a three week period. Reassessment showed that accuracy of plotting was considerably improved compared with existing charts (fig 2). Table 2 shows the objective performance of the new chart. There were improvements in detection rates of all parameters of physiological decline. This was significant in four of five parameters being measured: percentage improvements after chart redesign were, fever $16 \% \quad(p<0.05)$; hypotension $8 \% \quad(p>0.05)$; tachycardia $29 \% \quad(p<0.05)$; tachypnoea $41 \% \quad(p<0.05)$; hypoxia $45 \%(\mathrm{p}<0.05)$.

\section{DISCUSSION}

Modern medicine and the introduction of shorter working hours set by the New Deal and European Working Directive enforce time restraints on hospital practice. This means that clinicians may lose continuity of patient care. Nevertheless, clinicians need to be aware of patient deterioration. The bedside observation chart plays a critical part in identifying physiological decline of the ward patient.

Here we identify problems associated with poor chart design-a problem that is further compounded by nonstandardised recording practices.

Problems identified with existing charts were (fig 2A): temperature points are not always joined together as a graphical plot and writing in over the point obscures the value; charting of blood pressure and pulse together on the same overlain axes is easily correlated but difficult to read unless all the pulse values are joined together accuratelywriting in over the point also obscures the reading; individual points are often joined together as a curved rather than straight line; the percentage oxygen is not always recorded when measuring oxygen saturations; respiratory rate, the only parameter typically measured without an electronic machine is too frequently recorded as " 20 " suggesting approximation.

Change in respiratory rate and adequacy of oxygenation are early and important indicators of the deteriorating ward patient ${ }^{17815}$ but these parameters were among the worst detected by clinical staff: on average tachypnoea was missed by $19 \%$ of doctors and $63 \%$ of nurses; hypoxia was missed by $25 \%$ of doctors and $68 \%$ of nurses.

Table 2 Performance of new chart

\begin{tabular}{|c|c|c|c|c|c|c|c|c|c|c|}
\hline & \multicolumn{10}{|c|}{ Parameters of chart } \\
\hline & \multicolumn{2}{|l|}{ Fever } & \multicolumn{2}{|c|}{ Hypotension } & \multicolumn{2}{|c|}{ Tachycardia } & \multicolumn{2}{|c|}{ Tachypnoea } & \multicolumn{2}{|c|}{ Hypoxia } \\
\hline & A & B plotted & A & B plotted & A & $\begin{array}{l}\text { B plotted with BP } \\
\text { and written }\end{array}$ & A & $\begin{array}{l}\text { B plotted and } \\
\text { written }\end{array}$ & A & B plotted \\
\hline $\begin{array}{l}\text { Average detection } \\
\text { rate }(\%) \text {-cohorts } \\
1+2\end{array}$ & 84.0 & 100 & 82.5 & 90.5 & 69.5 & 98.5 & 58.0 & 98.5 & 53.5 & 98.5 \\
\hline
\end{tabular}




\section{Strengths and limitations of study}

- This study has scrutinised chart performance. The strength of the study lies with using an evidence based and multidisciplinary approach to observation chart redesign. To our knowledge this is the first such study in the United Kingdom. The methodology is therefore new and untested.

- The advantages of the new chart and implementation included increased detection of patient physiological decline, incorporated criteria for alerting clinical staff to decline, and standardisation across wards and potentially hospital trusts.

- We tried to avoid selection bias by testing all junior doctors and student nurses and healthcare assistants within our institution. The same participants within each cohort were tested before and after chart redesign.

- The sample size was small nevertheless statistical significance was shown.

- Although this is a single centre study it defines a significant problem with observation chart design that is unlikely to be unique to this centre alone.

On average the percentage detection rate of abnormalities was $35 \%$ higher for doctors compared with nurses. The reason for this is unknown and may represent local practices but raises the issue of the need for consolidation of skills and re-training in plotting observations and identifying abnormalities.

Overall, plotted parameters were better detected than written values ( $80 \%$ compared with $58 \%$ ) but in the case of respiratory rate, written values had higher rates of detection compared with graphical plots (65\% compared with 50\%).

Poor chart design can therefore seriously undermine the detection of patient deterioration and in this study we show the benefits that accrue from objectively quantifying chart performance.

Best features for making clinical staff aware of physiological decline based on objective and subjective testing were used to re-design a new observation chart that was standardised on all medical wards. Accurate detection of physiological deterioration is reliant on a chart that is well plotted and indeed in this study training of clinical staff in plotting observations based on defined standards resulted in significant improvements in the quality of the observation chart plot (compare fig $2 \mathrm{~A}$ and $2 \mathrm{~B}$ ) and in detection rates of most parameters. Parameters poorly identified on existing charts namely tachypnoea and hypoxia were significantly better detected on the new chart: there was a $41 \%$ and $45 \%$ increase in the detection rate of tachypnoea and hypoxia respectively by clinical staff. There were also significant percentage improvements in the detection rates of fever and tachycardia after intervention by $16 \%$ and $29 \%$ respectively.

Here for the first time we show that design of the bedside observation chart affects transfer of clinical data to staff. As a study confined to one hospital the problems identified with chart design were significant but unlikely to be unique to this centre.

With new working practices and the requirement of increased staff rotation, there may be a role for standardisation of observation charts nationally to increase ease of use and decrease the chance of clinical error. To this end the incorporation of physiological based early warning scores designed to alert nursing and medical staff to patients requiring closer supervision further decreases the chance of clinical error.

Anecdotal evidence suggests that chart re-design is all too often arbitrarily done by an individual or group of individuals perceived as having some experience in chart design. We show that an evidence based and multidisciplinary approach to observation chart redesign together with retraining in its use significantly improves detection rates of patient physiological decline.

We anticipate that this type of study could be extended to a national based project with the eventual aim of standardising an observation chart throughout the United Kingdom.

\section{ACKNOWLEDGEMENTS}

We acknowledge Olwyn Bamford for help with data collection and re-training of clinical staff on use of the new chart; Scott Wilson (UCLH) for statistical analysis; Brendan Curran for critically reading the manuscript.

\section{Authors' affiliations}

M T Chatteriee, J C Moon, D McCrea, Department of Medicine, North West London Hospitals NHS Trust (Central Middlesex Hospital), London, UK

R Murphy, Department of Nursing, North West London Hospitals NHS Trust (Central Middlesex Hospital)

Funding: none.

Competing interests: none declared.

Approval for this study was obtained from a clinical governance committee according to local practices.

\section{REFERENCES}

1 Goldhill DR. The critically ill: following your MEWS. Q J Med 2001;94:507-10

2 Mcquillan P, Pilkington S, Allan A, et al. Confidential inquiry into quality of care before admission to intensive care. BMJ 1998;316:1853-8.

3 Smith AF, Wood J. Can some in-hospital cardio-respiratory arrests be prevented? A prospective survey. Resusitation 1998;37:133-7.

4 Franklin C, Matthew J. Developing strategies to prevent inhopsital cardiac arrest: analysing responses of physicians and nurses in the hours before the event. Crit Care Med 1994;22:244-7.

5 Schein RM, Hazday N, Pena M, et al. Clinical antecedents to in-hospital cardiopulmonary arrest. Chest 1990;98:1388-92.

6 Buist MD, Jarmolowski E, Burton PR, et al. Recognising clinical instability in hospital patients before cardiac arrest or unplanned admission to intensive care. Med J Aust 1999;171:22-5.

7 Goldhill DR, White SA, Sumner A. Physiological values and procedures in the 24 hours before ICU admission from the ward. Anaesthesia 1999;54:529-34

8 Subbe CP, Davies RG, Williams E, et al. Effect of introducing the modified early warning score on clinical outcomes, cardio-pulmonary arrests and intensive care utilisation in acute medical admissions. Anaesthesia 2003;58:797

9 Lee A, Bishop G, Hillman KM, et al. The medical emergency team. Anaesth Intensive Care 1995;23:183-6.

10 Goldhill DR, Worthington L, Mulcahy A, et al. The patient-at risk team: identifying and managing seriously ill ward patients. Anaesthesia 1999;54:853-60.

11 Morgan RJM, Williams F, Wright MM. An early warning scoring system for detecting developing critical illness. Clin Intensive Care 1997;8:100.

12 Stenhouse C, Coates S, Tivey M, et al. Prospective evaluation of a modified early warning score to aid earlier detection of patients developing critical illness on a surgical ward. Br J Anaesth 2000;84:663.

13 Goldhill DR, McNarry AF. Physiological abnormalities in early warning scores are related to mortality in adult inpatients. Br J Anaesth 2004;92:784-6.

14 Subbe $C P$, Kruger $M$, Rutherford $P$, et al. validation of a modified early warning score in medical admissions. Q J Med 2001;94:521-6.

15 Sax FL, Charleson ME. Medical patients at high risk for catastrophic deterioration. Crit Care Med 1987;54:510-11. 